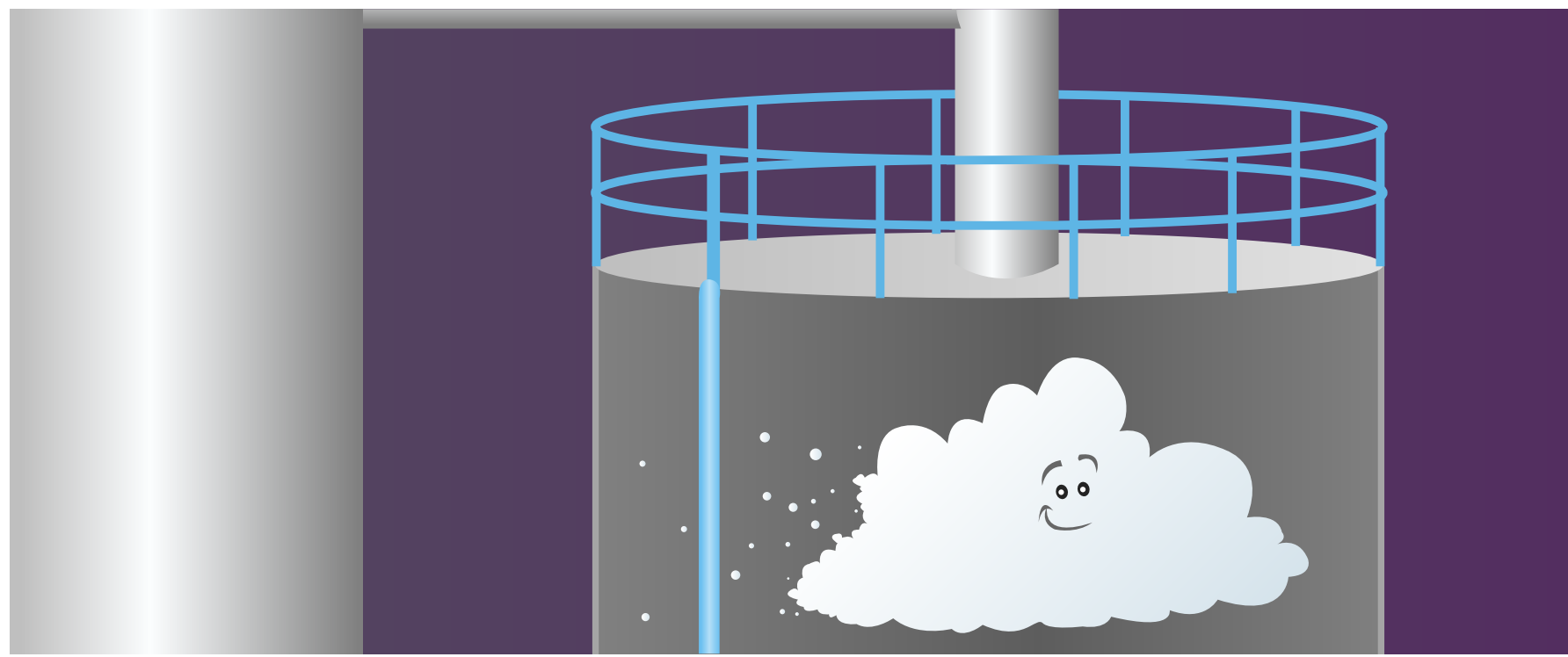

\title{
STUDYING THE SEEDS FOR CLOUDS AT THE CERN RESEARCH LABS
}

\section{Hamish Gordon*}

School of Earth and Environment, University of Leeds, Leeds, United Kingdom, European Organization for Nuclear Research (CERN), Geneva, Switzerland

\section{REVIEWED BY:}

BURY CHURCH OF ENGLAND HIGH SCHOOL 12-13 YEARS OLD
In the sky, tiny particles are needed for clouds to form. These particles can come directly from the ground (for example, from volcanoes or pollution released by industry) or they can form in the sky when sticky molecules clump together. More particles in the sky make clouds whiter. Whiter clouds reflect more of the sun's rays. This tends to cool down Earth's climate. So particles in the sky affect clouds and this in turn has an effect on global warming. Therefore, it is important to understand how these particles form. To study this, we measured how fast particles were made when we added controlled amounts of sticky gases to a tank at the CERN research lab. With computer simulations, we used the results to estimate which gases are most important for making particles in different parts of the atmosphere. This research will help scientists understand how particles affect Earth's climate.

\section{PARTICLES IN THE AIR}

Air is made up of the smallest objects we know about that are stable on their own: atoms and molecules. However, the air around us also carries many tiny particles in it: lumps of stuff too small to see. Like other solids 


\section{CLUSTER}

A small number of molecules that have stuck together. Clusters can grow into atmospheric particles if more molecules collide with and stick to the cluster. and liquids, the tiny particles in the air are made up of many molecules tied together by electrical attraction.

The particles are so light that they move with the air. Slowly, they fall to the ground because of gravity, but moving air pushes them around much more strongly than gravity pulls them down. The particles are blown around by the wind, and can travel long distances, even across continents, before making it down to Earth. When there are lots of particles in the air, they make the sky look hazy, so it gets harder to see long distances.

Close to land, the biggest particles in the air are usually dust or pollen. The smallest are clusters of just a few molecules, many times smaller than dust or pollen. Smoke is also made of particles. Smoke particles are bigger than the molecular clusters but smaller than dust. When particles come from human activity, we call them air pollution. However, air pollution can also be made of single molecules of poisonous gases.

Particles in the sky are important for two main reasons. First, breathing in too many of them is dangerous. For example, car exhaust fumes, like smoking, can cause cancer. The second reason particles are important is that they are needed for clouds to form.

\section{MAKING CLOUDS}

Clouds, whether they are big gray layers, fluffy white lumps, or streaks in the sky from aeroplanes, all have a few things in common. Most importantly, they are made up of tiny droplets of water, called "cloud droplets." Cloud droplets form when hot air from the Earth's surface rises and slowly cools down. Like the particles, the cloud droplets are so small that they do not fall to the ground (unless the cloud is raining). The rising air around them is pushing them up, gravity is pulling them down, and they end up just floating around the sky.

In the sky, unless there is some kind of surface for it to stick to, or unless it is really really cold $\left(-38^{\circ} \mathrm{C}\right)$, water exists as a gas. When there is a surface, water can liquefy (become liquid) or freeze onto the surface, as it does on the ground. However, in the sky, there are no big obvious surfaces. Therefore, to make a cloud droplet, you need a tiny particle, which has a small surface that water can stick to. The particle acts as the seed for the droplet. For droplet formation to work, the particles have to be about $50 \mathrm{~nm}$ across: about one thousandth of the thickness of a hair. Figure 1 shows where those particles in the atmosphere come from.

If there are fewer particles in the sky, there will be fewer droplets in each cloud. This makes the clouds darker. Dark things get hot more easily than bright things: for example, try (carefully) touching a black car when it is 


\section{FIGURE 1}

Where particles in the atmosphere come from (left and center of figure) and how they are lost (far right). Dust, sea spray, and smoke particles come directly from the ground (blue arrows), while gas molecules can also stick together to form particles (red arrows). If there are more particles in the sky, the clouds get whiter, so they reflect more of the Sun's rays back to space. Adapted from original diagram by Jasper Kirkby.

\section{GREENHOUSE GAS}

A gas in the atmosphere that absorbs energy from the Sun, for example carbon dioxide. Molecules containing more than two atoms are likely to be greenhouse gases.

\section{NUCLEATION}

In the context of this article, this means the formation of particles in the sky via gas molecules sticking together. Also called "secondary particle formation" or "new particle formation."

\section{CLOUD SEED}

Particle found in the atmosphere that water can condense onto to form a cloud droplet. Usually cloud seeds must be at least $50 \mathrm{~nm}$ in diameter.

\section{PRIMARY \\ PARTICLE}

A particle in the atmosphere that has come directly from the ground, instead of being formed by nucleation. Examples of primary particles often seen in the atmosphere include smoke (soot), dust, and sea salt.

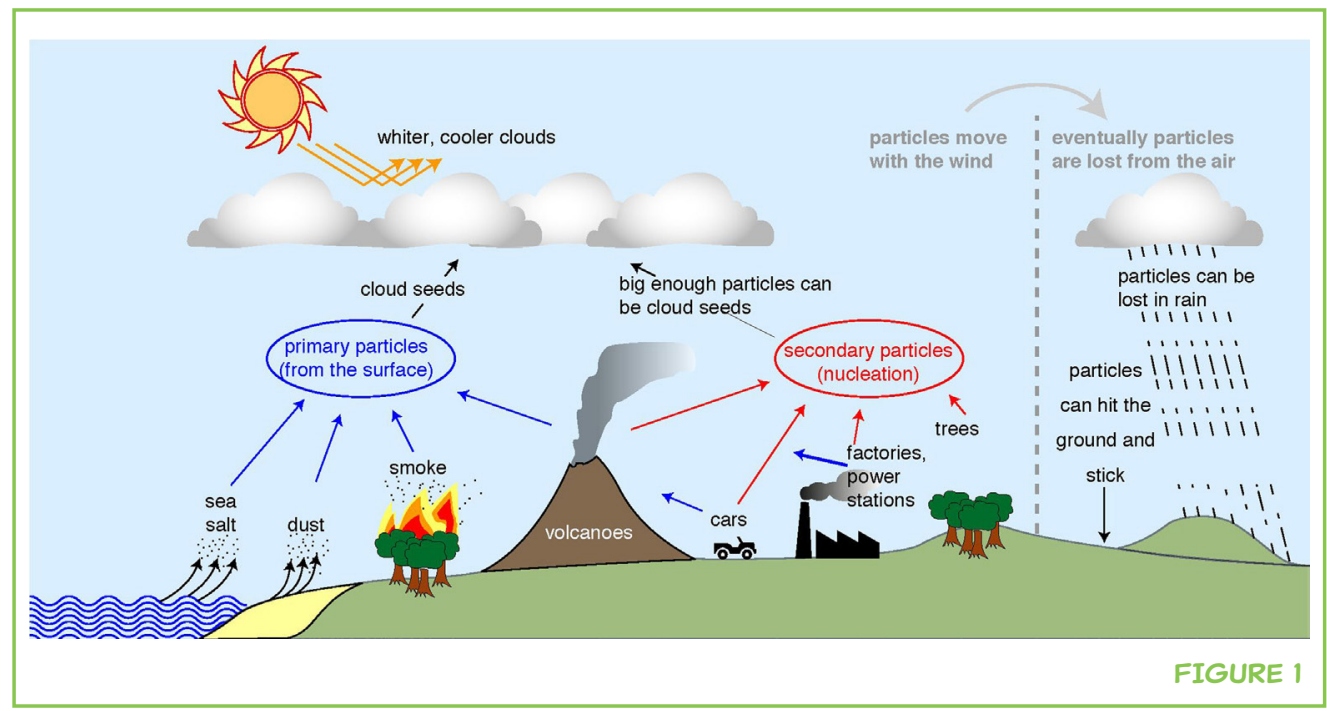

sunny, and then try touching a white car. Because darker clouds can get hot more easily, if clouds are darker, the Earth will warm up. This can have a big effect on the Earth's temperature, because, on average, clouds cover more than half of Earth's surface.

When we pollute the atmosphere, for example with smoke, we change the number of particles in the sky. This changes the brightness of clouds: they become whiter, reflecting more of the sun's rays and getting hot less easily. This has reduced the global warming from greenhouse gases like carbon dioxide that we have experienced over the last 100 years, perhaps by half, perhaps by a quarter. The effects of the particles on Earth's temperature are very uncertain. We need to understand these particles better to understand exactly what kind of warming (or cooling) they cause [1].

\section{MAKING PARTICLES}

Air is mostly made of nitrogen and oxygen molecules, but there are many other molecules floating around in the air. Some of the molecules that make up the air are stickier than others. (For a detailed explanation, see Box 1). Water is quite sticky, but some molecules are so sticky that they can make particles by sticking together on their own, without the surface that water needs. We call this process of sticking together nucleation.

As we saw in Figure 1, about half of the particles that act as cloud seeds in the sky come from the ground-like soot, dust, or pollen. We call these primary particles. The other half are made by nucleation, when the stickiest gases in the sky clump together [2]. The study we published recently is about which gases in the sky are sticking together to make the particles that act as the seeds of clouds. 


\section{BOX 1}

Why some molecules are stickier than others.

\section{Why some molecules stick together?}

I have described some molecules as "stickier" than others throughout this article. This actually means that some molecules are more strongly attracted to others by electrical forces. All atoms consist of a tiny nucleus and some electrons that sit outside the nucleus. How the electrons are arranged around the atom depends on what kind of atom it is [for example, in water $\left(\mathrm{H}_{2} \mathrm{O}\right)$, whether it is a hydrogen atom or an oxygen atom]. In molecules like water, the electrons are shared between the atoms. If the atoms in the molecule are the same [like in the oxygen molecule $\left(\mathrm{O}_{2}\right)$ ], the sharing is equal. If the atoms are different, usually some types of atom are better at attracting electrons toward them than others. In water, oxygen is better at pulling electrons toward itself than hydrogen, so the electrons mostly sit around the oxygen atom: the sharing is not equal.

Electrons have a negative electrical charge. Because most of the electrons in water are around the oxygen atom, not the hydrogen atoms, the oxygen atom ends up slightly negatively charged while the hydrogen atoms are slightly positive. The oxygen atom on one water molecule can attract the hydrogen atoms of another water molecule, because unlike charges attract. This makes water stickier than symmetric molecules like hydrogen $\left(\mathrm{H}_{2}\right)$ or oxygen $\left(\mathrm{O}_{2}\right)$, where the electrons are shared equally.

The stickier a molecule is, the more likely it is to exist as a liquid or solid instead of as a gas. This explains why water is liquid at room temperature, while oxygen and hydrogen are gases. Sulfuric acid and molecules made by terpenes (see later) are stickier even than water. In the atmosphere, they stick together first, and water sticks to them later to form cloud droplets.

Oxygen can take electrons from hydrogen, so they get a slight negative charge when they are in water

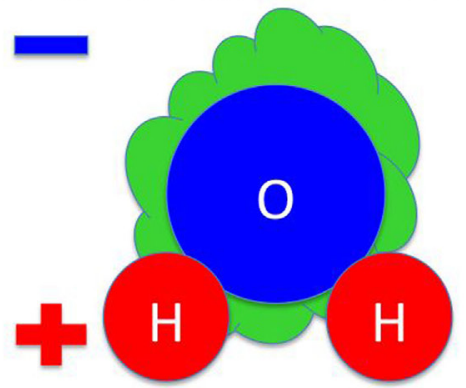

The green 'clouds' show (approximately!) where most of the outer electrons end up
Oxygen atoms in oxygen molecules have to share electrons equally, so stay neutral

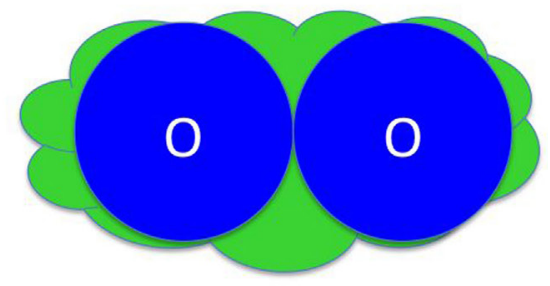

\section{WHAT WE KNEW ALREADY ABOUT MAKING PARTICLES}

Probably the best molecule for making particles in the sky is sulfuric acid. Sulfuric acid comes from volcanoes, plankton in the sea, or burning fossil fuels. Sulfuric acid molecules are very good at sticking to water. The molecules are also very good at sticking to each other. This means they can make particles in the sky and stick to water, to form the seeds of cloud droplets.

However, there is often not enough sulfuric acid around to make particles big enough to act as cloud seeds. A sulfuric acid molecule is only about half a nanometer across, so you need a lot of molecules to make a particle $50 \mathrm{~nm}$ across (big enough to be a cloud seed). And when two sulfuric acid molecules 
FIGURE 2

How molecules can stick together to become cloud seeds. When the first few molecules (on the left, shown as ball-and-stick drawings) clump together, the result is a cluster of molecules. In the cluster, dashed lines show the forces pulling the molecules together. This cluster is quite unstable and can easily fall apart again. If other molecules collide with the cluster, they can stick to it. If the cluster grows to a size where it is unlikely to fall apart, we call it a particle (red sphere). Larger particles can then act as the seeds for cloud droplets. The number of seeds can be reduced if small particles stick to larger ones (brown sphere). Adapted from original diagram by Jasper Kirkby.

\section{TERPENES}

Molecules emitted from trees, chemical formula $\mathrm{C}_{10} \mathrm{H}_{16}$. The reaction of these molecules with oxygen leads to molecules that form particles.

\section{COSMIC RAY}

High energy subatomic particles that hit the Earth from outer space.

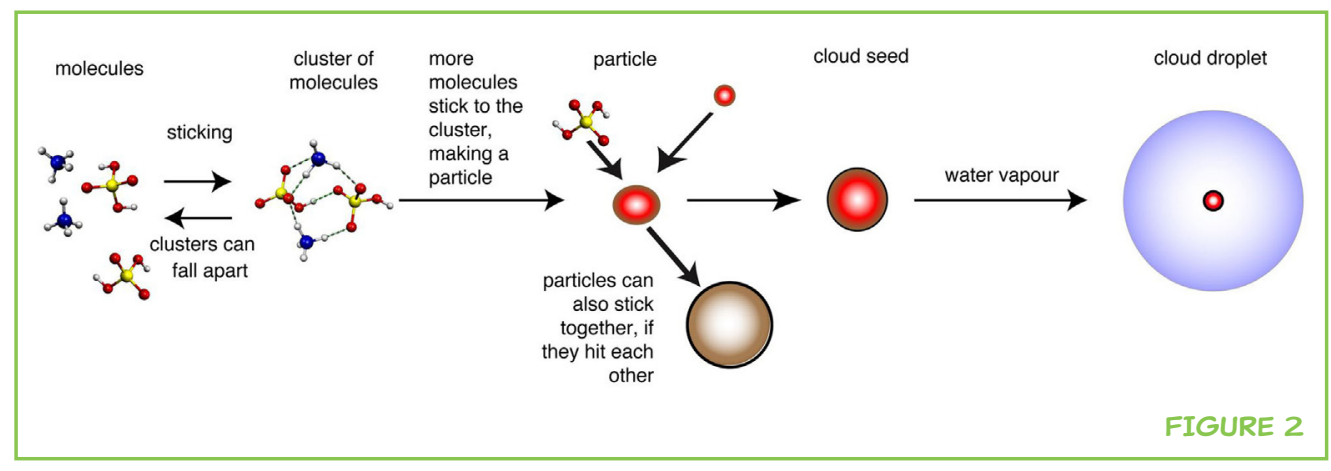

stick together, they can easily fall apart again. A cluster of two molecules is quite unlikely to grow into a cloud seed.

Some other molecules can stop the sulfuric acid molecules from falling apart by binding the little lump of sulfuric acid together. Sulfuric acid molecules can stick to each other, but there are other molecules that they stick to better. These molecules can stop the cluster from falling apart for long enough to let more sulfuric acid molecules hit it, stick to it and make it grow bigger. Bigger particles are much less likely to fall apart, because lots of the molecules are trapped inside the particle, so they cannot escape. This mechanism is shown in Figure 2.

\section{OUR STUDY: WHAT IS STICKING THE SULFURIC ACID MOLECULES TOGETHER?}

A good glue for sulfuric acid molecules is ammonia [3]. Like sulfuric acid, ammonia is poisonous in large amounts, but you have a little of it in your body. It causes the smell of urine. From the data we collected in our study, we estimated that almost two-thirds of the particles in the atmosphere that were made from sulfuric acid also needed ammonia to glue the acid molecules together.

Other good glues for sulfuric acid molecules come from trees [4]. Trees, particularly pine trees, give out molecules that we call terpenes, which are found in paint-stripper (turpentine). Terpenes also cause pine forests to smell fresh-much nicer than urine! After some chemical reactions with other things in the air these molecules also make good glue. From our study, we estimated that about one in five particles in the atmosphere were formed with terpenes as the glue. And in some cases, molecules related to terpenes are not just good glue, they can make particles without sulfuric acid at all [5].

There is one last thing we studied that is important for making particles in the sky: "cosmic rays." These are subatomic (really, really small) particles from outer space that are hitting the Earth all the time. Cosmic rays can collide with sulfuric acid or other molecules in the atmosphere and make them stickier. They do this by knocking electrons out of the molecules they collide with, 


\section{FIGURE 3}

The CLOUD experiment at CERN. The tank (center of photo) is covered by insulation to keep the temperature from changing. The concrete blocks (background) keep subatomic particles inside, away from scientists working in the hall. Photo by Antti Onnela.

\section{CERN}

European Organisation for Nuclear Research, home of the CLOUD experiment and several other experiments involving subatomic particles. The largest experiments at CERN use subatomic particles from the Large Hadron Collider (LHC).

\section{SUBATOMIC PARTICLE}

Particles smaller than atoms. The most common subatomic particles are protons, neutrons, and electrons. These make up atoms. Cosmic rays can contain more exotic subatomic particles, for example pions or muons.

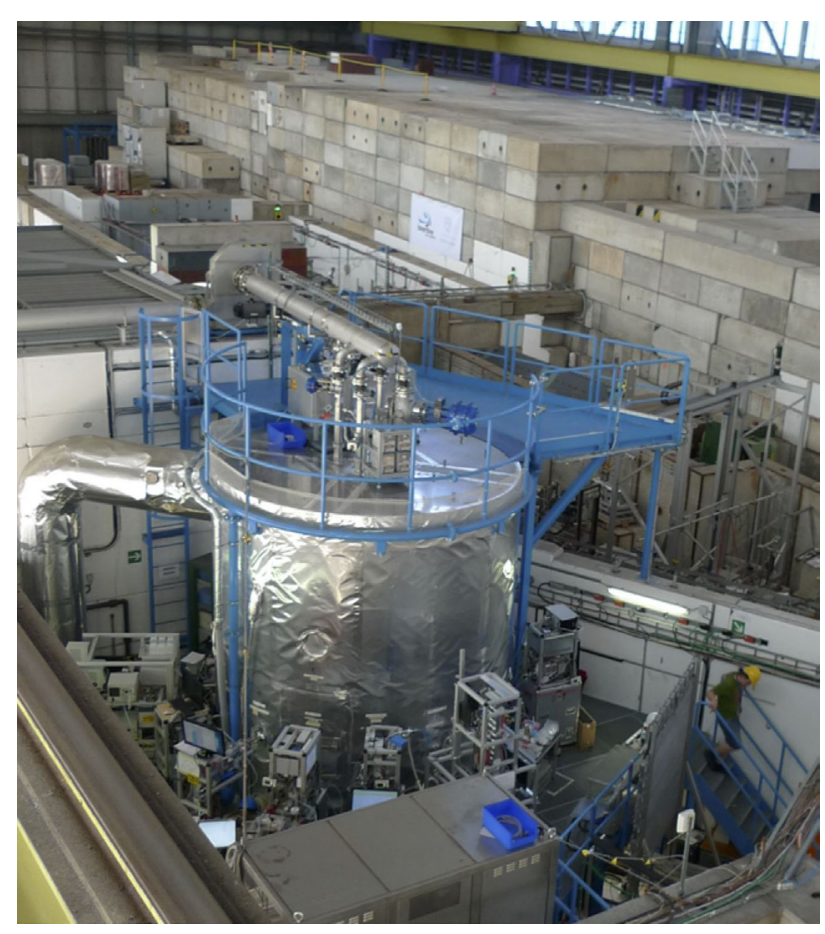

FIGURE 3

leaving them positively charged. The electrons can then stick to other molecules, making them negative. Just as like charges attract, electrically charged molecules can also attract each other inside a particle. This can help particles stay together without falling apart [6]. Our study allowed us to estimate, better than ever before, how important cosmic rays are in particle formation in the atmosphere.

\section{HOW DID WE FIND ALL THIS OUT?}

Scientists usually test their ideas by doing experiments. We have a special laboratory in Switzerland where we can do experiments to make particles. It is at a large research center called CERN, the same place as the Large Hadron Collider (LHC). The LHC is a much bigger experiment that smashes subatomic particles (very similar to cosmic rays) together. Scientists at the LHC do this to try to learn about how the Universe started and what is inside the atoms we are made of. Our smaller experiment is called CLOUD, which stands for Cosmics Leaving Outdoor Droplets.

The CLOUD experiments [1] (http://cloud.web.cern.ch/) take place in a big metal tank, about $3 \mathrm{~m}$ high and wide, that is filled with air. In the tank (Figure 3), we mix gases that we think will make particles and measure how fast the particles appear. In the atmosphere, there are all kinds of gases, and it is hard to figure out which gases are making particles and which are not.

Our tank is special because it is extremely clean: our air is made artificially by mixing liquid nitrogen and liquid oxygen, and it is sealed so no pollution 
FIGURE 4

Flowchart explaining the computer simulation needed to turn experiments at CLOUD into predictions about the effects of particle formation on the Earth's climate. The computer program puts together our results about how well molecules stick together with estimates of how much sticky gas there is at each place in the atmosphere. This lets us figure out how many particles form in a certain amount of time in each part of the atmosphere. Then, we calculate how many particles are lost in that time, as they stick together or fall to the ground. Balancing particle formation and loss lets us work out how many particles there are in each part of the atmosphere. We can then see how adding particles might affect the brightness of the clouds in that place: more cloud seeds leads to brighter clouds. With this, we can see how much of the Sun's energy will be reflected, and what it all means for Earth's climate.

\section{ALTITUDE}

Height above Earth's surface.

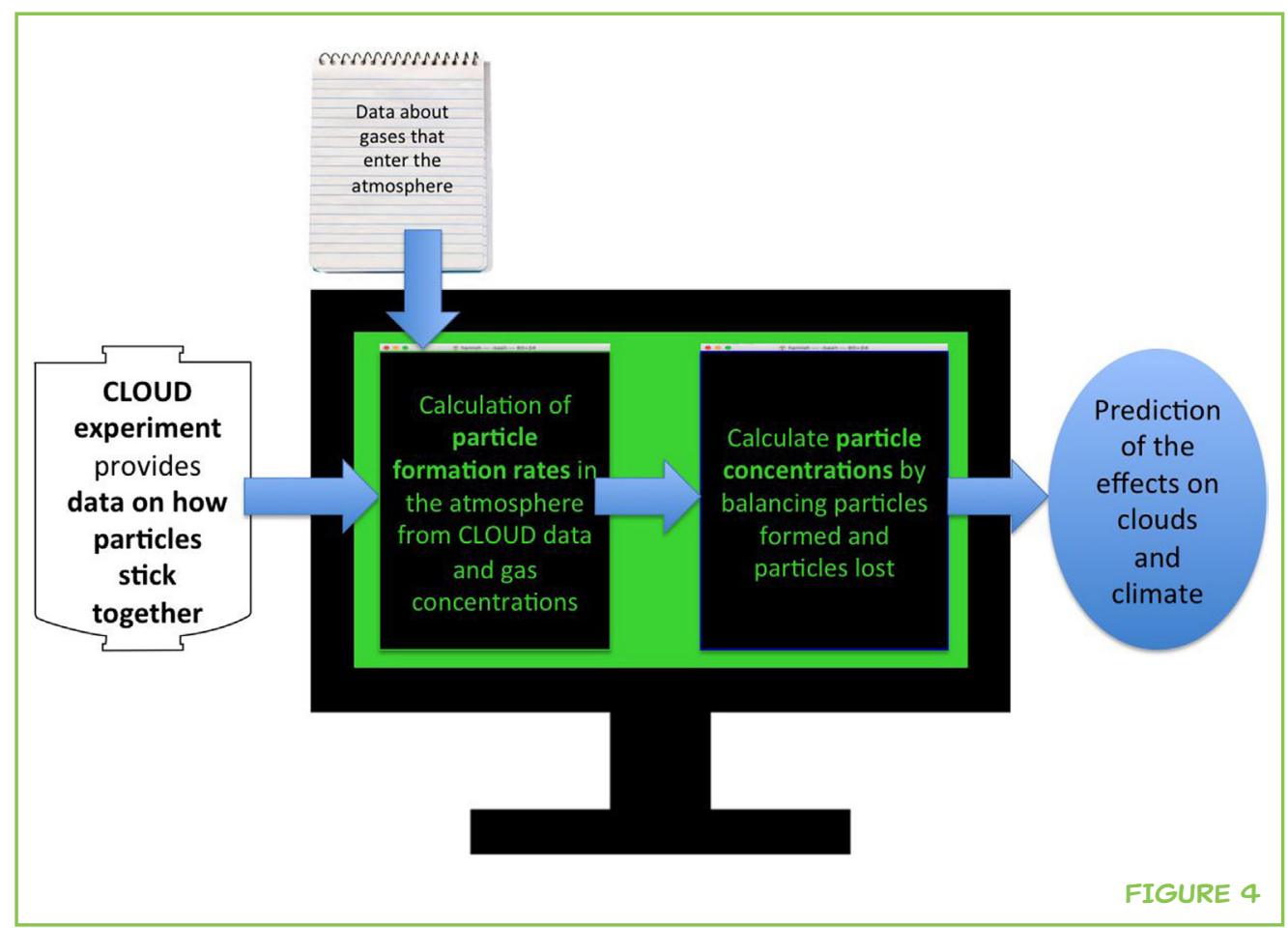

from outside can get in. We only put in a few gases, and we know exactly how much of each gas we put in. This allows us to measure how sticky each gas is.

Using CLOUD, we can also see how cosmic rays help to make cloud-seeding particles, by sending much smaller subatomic particles generated from CERN's other experiments into our tank. This has the same effect as sending cosmic rays through our tank. Like cosmic rays, the subatomic particles make the molecules stickier. We can see how many more particles we make when we switch on these artificial cosmic rays, and this helps us understand the difference the cosmic rays are making in particle formation.

In the atmosphere, the number of cosmic ray particles is higher at high altitude (on aeroplanes or mountain-tops, for example) than it is at ground level, because they get absorbed by the air. Therefore, by turning on the extra particles from the other CERN experiments, we can replicate conditions high in the atmosphere. This is the main reason why the CLOUD experiment needs to be at CERN. Once we have measured how well the gases stick together in the CLOUD experiment, we use the results in a computer simulation of particles in the atmosphere. This is a set of mathematical recipes for all the processes in Figure 1, written up in a big computer program [7]. This program (Figure 4) tells us the amount of sticky gas at all points in the lower atmosphere (altitude below about $20 \mathrm{~km}$ ). With this data about the amounts of gases, we can then use the results from the CLOUD experiments to tell us how many particles will form in a second, or in a day. Once we have the particles in our simulation, we model how they grow or stick together to make cloud seeds, and how they are lost to the ground. This tells us how important the different ways particles can form are at all points in the atmosphere. 


\section{WHAT DOES IT ALL MEAN?}

Two hundred years ago, there was about half as much sulfuric acid in the atmosphere as there is now. This is mostly because we have burned so much coal in the past two hundred years. So, what makes cloud seeds now could be quite different from what made cloud seeds two hundred years ago. Before we started burning lots of coal, release of terpenes from trees must have been more important, and sulfuric acid less important [8].

In the future, therefore, as we stop burning dirty coal, release of terpenes from trees will become more important once again. In some places, we will have less acid to make cloud seeds, and there will be fewer cloud seeds overall. Clouds will probably have the same amount of water in them, but fewer droplets. This can make them more likely to release their water as rain. It also makes the clouds darker, leading to warming of the planet, as we already discussed [1]. Our study gives an estimate of how important molecules from trees are, compared with ammonia, for making cloud seed particles. This information will help scientists to estimate how much of a difference cleaning the air will make to our climate.

\section{ORIGINAL SOURCE ARTICLE}

Dunne, E. M., Gordon, H., Kürten, A., Almeida, J., Duplissy, J., Williamson, C., et al. 2016. Global atmospheric particle formation from CERN CLOUD measurements. Science 354:1119-24. doi:10.1126/science.aaf2649

\section{REFERENCES}

1. Stocker, T. F., Qin, D., Plattner, G-K., Tignor, M., Allen, S. K., and Boschung, J., et al., eds. 2013. Climate Change 2013: The Physical Science Basis. Contribution of Working Group I to the Fifth Assessment Report of the IPCC. Cambridge, UK: Cambridge University Press.

2. Merikanto, J., Spracklen, D. V., Mann, G. W., Pickering, S. J., and Carslaw, K. S. 2009. Impact of nucleation on global CCN. Atmos. Chem. Phys. 9:8601-16. doi:10.5194/acp-9-8601-2009

3. Kulmala, M., Pirjola, U., and Mäkelä, J. M. 2000. Stable sulphate clusters as a source of new atmospheric particles. Nature 404:66-9. doi:10.1038/35003550

4. Went, F. W. 1960. Blue hazes in the atmosphere. Nature 187:641-3. doi:10.1038/187641a0

5. Kirkby, J., Duplissy, J., Sengupta, K., Frege, C., Gordon, H., and Williamson, C., et al. 2016. Ion-induced nucleation of pure biogenic particles. Nature 533:521-6. doi:10.1038/nature17953

6. Kirkby, J., Curtius, J., Almeida, J., Dunne, E., Duplissy, J., and Ehrhart, S., et al. 2011. Role of sulphuric acid, ammonia and galactic cosmic rays in atmospheric aerosol nucleation. Nature 476:429-33. doi:10.1038/nature10343 
7. Spracklen, D. V., Pringle, K. J., Carslaw, K. S., Chipperfield, M. P., and Mann, G. W. 2005. A global off-line model of size-resolved aerosol microphysics: I. Model development and prediction of aerosol properties. Atmos. Chem. Phys. 5:2227-52. doi:10.5194/acp-5-2227-2005

8. Gordon, H., Sengupta, K., Rap, A., Duplissy, J., Frege, C., and Williamson, C., et al. 2016. Reduced anthropogenic aerosol radiative forcing caused by biogenic new particle formation. Proc. Natl. Acad. Sci. U.S.A. 113:12053-8. doi:10.1073/ pnas. 1602360113

SUBMITTED: 17 March 2017; ACCEPTED: 19 July 2017;

PUBLISHED ONLINE: 04 August 2017.

EDITED BY: Berend Smit, École Polytechnique Fédérale de Lausanne, Switzerland

CITATION: Gordon H (2017) Studying the Seeds for Clouds at the Cern Research Labs. Front. Young Minds 5:43. doi:10.3389/frym.2017.00043

CONFLICT OF INTEREST STATEMENT: The author declares that the research was conducted in the absence of any commercial or financial relationships that could be construed as a potential conflict of interest.

COPYRIGHT @ 2017 Gordon. This is an open-access article distributed under the terms of the Creative Commons Attribution License (CC BY). The use, distribution or reproduction in other forums is permitted, provided the original author(s) or licensor are credited and that the original publication in this journal is cited, in accordance with accepted academic practice. No use, distribution or reproduction is permitted which does not comply with these terms.

\section{REVIEWED BY}

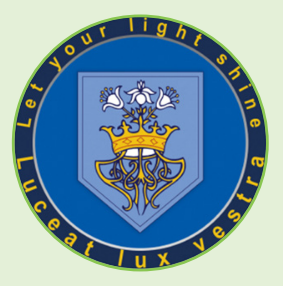

\section{BURY CHURCH OF ENGLAND HIGH SCHOOL, 12-13 YEARS OLD}

We are a High School located in Bury, Greater Manchester, England. The pupils who took part in this review were a class of 31 , ages ranging from 12- to 13 years. The pupils at Bury Church of England are keen and enthusiastic scientists and really enjoyed learning how clouds are formed in the atmosphere. They were also really excited by the experimental methods carried out at CERN. The review project was carried out with help of the science mentor, Lyndsey Ayres, MChem.

\section{AUTHOR}

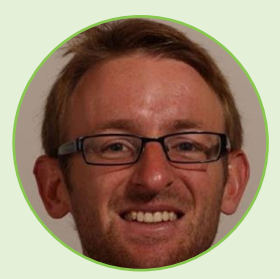

\section{HAMISH GORDON}

I am a physicist working at the University of Leeds. I help to develop computer models that simulate particles and clouds in the atmosphere, with other scientists at Leeds, the UK Met Office and at CERN's CLOUD experiment. We try to understand how airborne particles from natural sources or from pollution can affect clouds, and what the effects mean for Earth's climate. When I am not at my desk I like cooking, running, or exploring Yorkshire on my road bike. *hamish.gordon@cern.ch 